\title{
Long-Term Antithyroid Drug Treatment of Patients With Graves' Disease
}

\section{David S. Cooper}

Division of Endocrinology, Diabetes, and Metabolism, Department of Medicine, Johns Hopkins University, School of Medicine, Baltimore, Maryland, U.S.A.

Review of: Bandai S, Okamura K, Fujikawa M, Sato K, Ikenoue H, Kitazono T 2019 The long-term followup of patients with thionamide-treated Graves' hyperthyroidism. Endocr J. Epub 2019 Mar 28. PMID: 30918165.

\section{SUMMARY}

\section{Background}

Antithyroid drugs remain an important treatment for hyperthyroidism due to Graves' disease (1). There are two main therapeutic protocols that are used; in the first scenario, antithyroid drugs are given for 12 to 18 months, and, if serum anti-TSH receptor antibody (TRAb) levels have normalized, the drug is discontinued and patients are monitored for recurrence. If antibodies persist, however, definitive treatment with radioiodine or surgery may be recommended $(2,3)$, although continued antithyroid drug therapy is also reasonable in patients with well-controlled mild disease $(2,3)$. In the second scenario, antithyroid drugs are given for as long as necessary, until TRAbs disappear. This could be for years or even for a lifetime $(4,5)$. The aim of the current study was to describe long-term outcomes in 549 adult patients with Graves' disease treated with antithyroid drugs for $>8$ years and to relate remission and final outcomes to patterns of serum TRAb titers (6).

\section{Methods}

This retrospective study included 549 patients (mean age, 36-41 years) with untreated Graves' disease seen at the Kyushu University Hospital, Fukuoka, Japan, between 1981 and 2001. The patients were followed from 8 to 36 years. Greater than $90 \%$ of patients were treated with methimazole. The patients were classified into four groups after follow-up: Group AO were TRAb-negative at the outset, Group A2 became TRAb-negative within 2 years of methimazole treatment, Group A5 became TRAb-negative after 2 to 5 years of treatment (all combined into Group A), and Group B, in whom TRAbs remained positive after $>5$ years of continuous treatment. In Group A, following the disappearance of TRAb and normalization of other clinical parameters (e.g., goiter), antithyroid drug therapy was stopped. If patients remained euthyroid with normal thyroid function for $>1$ year, they were considered to be in remission. Otherwise, they were classified as "nonremission"; this latter group also included patients who were treated with ablative therapy.

\section{Results}

Serum TRAb was negative at baseline in 52 (9.5\%) of patients, despite a high 24-hour radioiodine uptake (Group A0). Among the remaining 497 patients who were TRAb-positive at baseline, TRAb became negative after 2 years of continuous therapy in 274 patients (49.9\%) and after 2 to 5 years in an additional 107 patients (19.5\%), for a total of 433 patients (78.9\%) (Groups A2 and A5). However, in approximately $50 \%$ of Group A patients whose TRAb became negative, TRAb levels subsequently became positive again (Group 1A); in these patients ( $n=226$ ), whose TRAb course was called "fluctuat- 
ing," the rates of remission at the end of follow-up were significantly lower than in the group whose TRAb levels remained normal $(n=201)(37.2 \%$ vs. $88.9 \%, \mathrm{P}<0.0001)$. A third group of patients ( $\mathrm{n}=$ $116,21.1 \%$ of patients) had a "smoldering" course, with TRAb levels remaining positive during continuous antithyroid drug therapy for $>5$ years (Group B). However, even in this group, TRAb gradually declined and became negative after $>5$ years in 23 patients (19.8\% of the "smoldering" group).

Overall, the time to remission for all groups combined was a median of 6.8 years (interquartile range, 4.0-10.9). The cumulative fraction going into remission was $18.2 \%$ after 5 years, 38.3\% after 10 years, $41.8 \%$ after 15 years, and $52.1 \%$ after 20 years. Approximately $6 \%$ of patients became spontaneously hypothyroid over the $>8$-year follow up period. Overall, about $20 \%$ of patients received ablative therapy $(2.2 \%$ of those in Group 1, 28\% of those in Group 1A, and $40.5 \%$ of those in Group B).
Using multivariate logistic regression analysis, baseline factors predicting failure to remit were male sex and younger age, but the degree of thyroid dysfunction, thyroid volume, or TRAb levels were not predictive. However, there were factors during antithyroid drug therapy that were predictive of failure to remit, including time to normalization of TRAb and persistence of a palpable goiter. There was no difference in remission rates between patients treated with methimazole versus PTU or the dose of antithyroid drug employed.

\section{Conclusions}

In patients with Graves' disease, remissions often occur after 4 to 11 years of antithyroid drug therapy. Some patients have resolution of TRAb within 2 to 5 years, while in others, TRAb levels fluctuate or remain positive for extended periods ( $>5$ years). In this cohort, the odds of remission could not be predicted by baseline clinical or laboratory variables. In patients hoping to avoid ablative therapy, continuous antithyroid drug therapy for $>5$ years is reasonable.

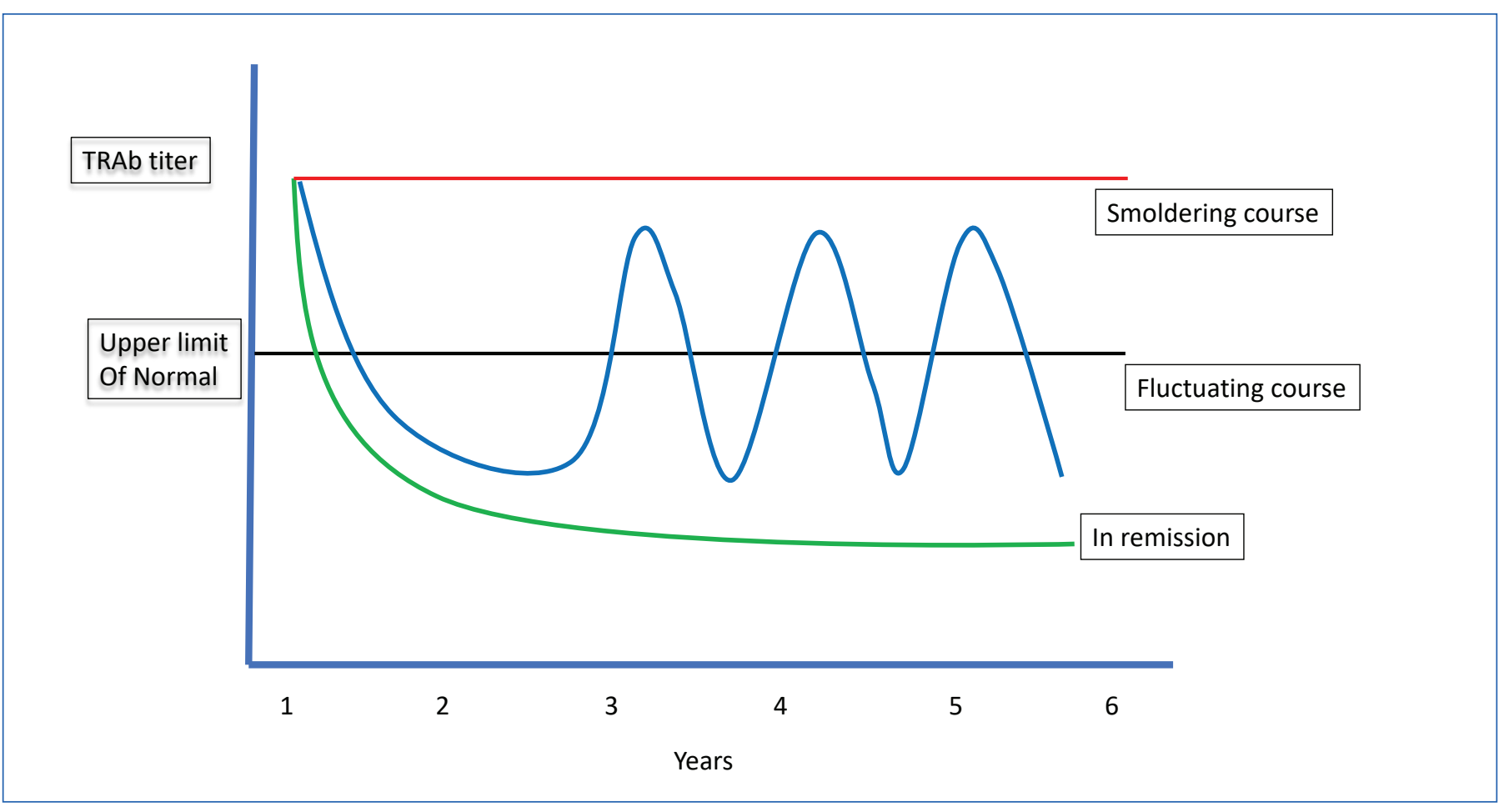



Patients With Graves' Disease

\section{COMMENTARY}

This cohort of antithyroid-drug-treated adult Graves' disease patients is one of the largest reported in the literature and has one of the longest follow-up periods. The article presents new information regarding patterns of disappearance of serum TRAb concentrations over time, which appear to have an impact on the probability of remission. Unlike other studies, which found useful predictors of remission in baseline clinical and laboratory tests (e.g., 7,8), the authors of this report found no baseline parameter predicting the likelihood of remission.

When asked about the management of prototypical Graves' disease, surveys of clinicians indicate that the majority would continue antithyroid drug therapy for 12 to 24 months $(9,10)$. The 2016 American Thyroid Association clinical practice guidelines for the management of hyperthyroidism state that after 12 to 18 months of antithyroid drug therapy: "Patients with persistently high TRAb could continue ATD therapy... or opt for alternate definitive therapy with RAl or surgery. In selected patients (i.e., younger patients with mild stable disease on a low dose of $\mathrm{MMI})$, long-term $\mathrm{MMI}$ is a reasonable alternative approach" (2). The present article confirms data from other series of adult (5) and pediatric (11) patients that show that remissions in Graves' disease patients continue to be achieved after many years $(>5-10)$ of continuous antithyroid drug therapy. Therefore, in patients who wish to avoid permanent hypothyroidism or potential complications of ablative therapy, long-term antithyroid drug therapy is a viable option.

However, in older patients (e.g., those >60 years of age), definitive therapy should be more strongly considered at the time of initial diagnosis or if TRAb titers persist for more than 1 to 2 years of antithyroid drug therapy (3). This is because the effects of persistent or recurrent hyperthyroidism that could develop are potentially life-threatening (e.g., atrial fibrillation or other adverse cardiovascular outcome (12)) or clinically significant (e.g., osteoporosis). While it is true that older patients are more likely to achieve remission (7), the worry that remissions are not necessarily lifelong makes definitive treatment more reasonable (3). However, in young and middle-aged patients, long-term therapy with methimazole may become a more widely accepted strategy, especially given recent data on the adverse effects of radioiodine therapy on quality of life (13). Failure to attain normal TRAb levels after 12 to 18 months of methimazole therapy does not rule out the possibility of remission occurring over a longer time horizon of 5 to 10 years. Even in patients with "smoldering" TRAb, about $20 \%$ became negative over prolonged follow-up.

\section{References}

1. Burch HB, Cooper DS 2018 Anniversary review: Antithyroid drug therapy: 70 years later. Eur J Endocrinol 179:R261-R274.

2. Ross DS, Burch HB, Cooper DS, Greenlee MC, Laurberg P, Maia AL, Rivkees SA, Samuels M, Sosa JA, Stan MN, Walter MA 20162016 American Thyroid Association guidelines for diagnosis and management of hyperthyroidism and other causes of thyrotoxicosis. Thyroid 26:1343-1421.

3. Kahaly GJ, Bartalena L, Hegedüs L, Leenhardt L, Poppe K, Pearce SH 20182018 European Thyroid Association guideline for the management of Graves' hyperthyroidism. Eur Thyroid J 7:167-186. 
4. Howard JE 1967 Treatment of thyrotoxicosis. JAMA 202:706-709.

5. Azizi F, Ataie L, Hedayati M, Mehrabi Y, Sheikholeslami F 2005 Effect of longterm continuous methimazole treatment of hyperthyroidism: comparison with radioiodine. Eur J Endocrinol 152:695-701.

6. Bandai S, Okamura K, Fujikawa M, Sato K, Ikenoue $\mathrm{H}$, Kitazono T 2019 The long-term followup of patients with thionamide-treated Graves' hyperthyroidism. Endocr J. Epub 2019 Mar 28.

7. Vos XG, Endert E, Zwinderman AH, Tijssen JG, Wiersinga WM 2016 Predicting the risk of recurrence before the start of antithyroid drug therapy in patients with Graves' hyperthyroidism. J Clin Endocrinol Metab 101:1381-1389.

8. Struja T, Fehlberg H, Kutz A, Guebelin L, Degen C, Mueller B, Schuetz P 2017 Can we predict relapse in Graves' disease? Results from a systematic review and meta-analysis. Eur J Endocrinol 176:87-97.

9. Burch HB, Burman KD, Cooper DS $2012 \mathrm{~A}$ 2011 survey of clinical practice patterns in the management of Graves' disease. J Clin Endocrinol Metab 97:4549-4558.
10. Bartalena L, Burch HB, Burman KD, Kahaly GJ 2016 A 2013 European survey of clinical practice patterns in the management of Graves' disease. Clin Endocrinol (Oxf) 84:115-120.

11. Léger J, Gelwane G, Kaguelidou F, Benmerad M, Alberti C 2012 French Childhood Graves' Disease Study Group. Positive impact of long-term antithyroid drug treatment on the outcome of children with Graves' disease: national long-term cohort study. J Clin Endocrinol Metab 97:110-119.

12. Okosieme OE, Taylor PN, Evans C, Thayer D, Chai A, Khan I, Draman MS, Tennant B, Geen J, Sayers A, et al. 2019 Primary therapy of Graves' disease and cardiovascular morbidity and mortality: a linkedrecord cohort study. Lancet Diabetes Endocrinol 7:278-287.

13. Törring O, Watt T, Sjölin G, Byström K, AbrahamNordling M, Calissendorff J, Cramon PK, Filipsson Nyström H, Hallengren B, Holmberg M, et al. 2019 Impaired quality of life after radioiodine therapy compared to antithyroid drugs or surgical treatment for Graves' hyperthyroidism: a long-term follow-up with the Thyroid-Related Patient-Reported Outcome Questionnaire and 36-Item Short Form Health Status Survey. Thyroid 29:322-331. 